\title{
Young Stars in the Outer Disc of NGC 6822
}

\author{
W.J.G de Blok \\ Dept of Physics and Astronomy, Cardiff University, 5 The Parade, \\ Cardiff, CF24 3YB, UK \\ F. Walter \\ NRAO AOC, PO Box O, 1003 Lopezville Road, Socorro, NM \\ 87801-0387, USA
}

\begin{abstract}
We present wide-field optical imaging covering the entire neutral hydrogen disc of the Local Group dwarf galaxy NGC 6822. These observations reveal the presence of numerous young stars at large galactocentric radii. The extended distribution of young stars implies that stars do form in the outer disks of dwarf galaxies; the HI is not necessarily much more extended than the stellar population. This finding has important consequences for the chemical enrichment of the interstellar medium throughout dwarf galaxies.
\end{abstract}

NGC 6822 is the most nearby (490 kpc) late-type dwarf galaxy outside the Milky Way/LMC/SMC system and therefore a prime target to study in detail the interplay between of star formation and the atomic interstellar medium (ISM). Observations in the 21-cm line of neutral hydrogen by de Blok \& Walter (2000) and Weldrake at al (2003) show that the galaxy is dark matter dominated, gas rich and reveal many interesting features such as a highly structured HI disk, a supergiant HI shell and a companion cloud. Here we discuss the properties and distribution of the young stellar populations.

The entire extent of the HI disk was observed in $B$ and $R$ with the 40 " at Siding Spring, Australia, in July 1999. The DOPHOT package was used to create a catalog of stellar objects in the field. We extracted the blue stars $(B-R<0.75)$ from our catalogue. Their colours and luminosities are consistent with those of main sequence stars of types between O5 $\left(M_{B} \sim-5.8\right)$ and B5 $\left(M_{B} \sim-1.3\right)$. Their main sequence lifetimes vary between $\sim 1 \mathrm{Myr}$ or less and $\sim 80$ Myr, respectively.

Figure 1 shows our main results. Panels $a$ and $b$ show the distribution of blue stars on the sky and compared to the HI distribution. The blue stars cover a much larger area than what is usually regarded as the optical component of NGC 6822. The distribution of blue stars is as extended as that of the inner main HI disk. Stars are also present in the NW HI cloud. Panel c shows the distribution of Carbon-stars taken from Letarte et al (2000). C-stars trace the intermediate age (3-5 Gyr) population. The distribution of young stars is very different from that of the older population.

The presence of blue stars all over the main body of NGC 6822 indicates that star formation is not just occurring in the optical disk, but that pollution of 
the ambient ISM is taking place on a larger scale. In summary, the distribution of the atomic ISM in dwarf galaxies is not necessarily more extended than that of its stellar population.

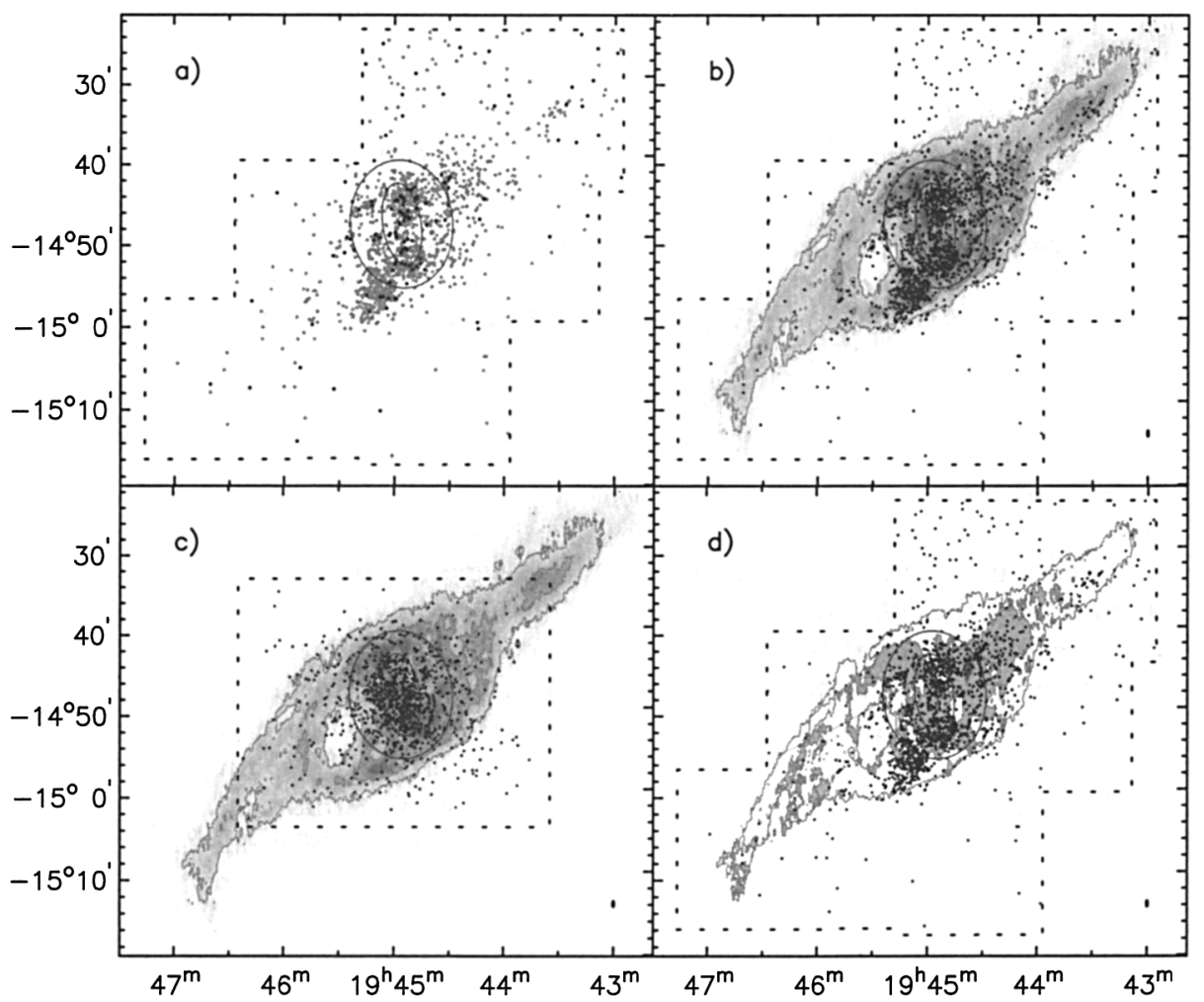

Figure 1. Distribution of stars in NGC 6822. Dotted lines indicate the survey area. Outer ellipse: $D_{25}\left(15.5^{\prime} \times 13.5^{\prime}[\mathrm{RC} 3]\right)$, inner ellipse: optical bar. a) Distribution of blue stars. Grey dots: $B-R<0.75$ and $m_{R}>19$; black dots: $B-R<0.75$ and $m_{R} \leq 19$. b) Blue stars on HI surface density map. Contours: $\sigma=(2.5 ; 5) \cdot 10^{20} \mathrm{~cm}^{-2}$ (inclination corrected). c) As b), with distribution of C stars (Letarte et al 2000). d) Blue stars on velocity dispersion map. White: dispersion $\delta<8$ $\mathrm{km} / \mathrm{s}$; light-grey: $8 \leq \delta<12 \mathrm{~km} / \mathrm{s}$; dark grey: $12 \leq \delta<16 \mathrm{~km} / \mathrm{s}$.

\section{References}

de Blok, W.J.G., Walter, F. 2000, ApJ, 537, L95

Letarte, B., Demers, S., Battinelli, P., Kunkel, W.E. 2002, ApJ, 123, 832

Weldrake, D.T.F., de Blok, W.J.G., Walter, F. 2003, MNRAS, 340, 12 